\title{
Nuevos registros de hongos gasteroides leñosos desertícolas (Agaricaceae): Battarrea stevenii (Liboschitz) Fr. y Chlamydopus meyenianus (Klotzsch) Lloyd, en el norte de Chile
}

\author{
(New reports of woody desert gasteroid fungi (Agaricaceae): Battarrea steve- \\ nii (Liboschitz) Fr. and Chlamydopus meyenianus (Klotzsch) Lloyd, \\ in northern Chile.)
}

Claudina Jirón A.*

Fundación R.A. Philippi de Estudios Naturales. Corte de Apelaciones 1051. Depto. 403 Vitacura, Santiago, Chile *cjironal@yahoo.com

RECIBIDO: 31 de Agosto de 2016 APROBADO:07 de Noviembre de 2016

DOI: 10.22370/bolmicol.2016.31.2. 455

LA AUTORA DECLARA NO TENER CONFLICTO DE INTERESES

Palabras clave: hongo gasteroide leñoso desertícola

Key words: woody desert gasteroid fungi

\section{RESUMEN}

Battarrea stevenii (Liboschitz) Fr. y Chlamydopus meyenianus (Klotzsch) Lloyd son dos hongos gasteroides raramente recolectados en Chile. Habitan regiones áridas y emergen después de las lluvias de primavera. Nuevas localidades y descripciones se presentan aquí.

\section{INTRODUCCIÓN}

Los hongos gasteroides son un grupo de basidiomicetos que forman cuerpos fructíferos consistentes en una masa de esporas (gleba) encerrada dentro de una capa externa (peridio). Tradicionalmente se ubicaban en la Clase Gasteromycetes pero en base a estudios moleculares se han determinado ancestros agaricoides por lo cual se
ABSTRACT

Battarrea stevenii (Liboschitz) Fr. and Chlamydopus meyenianus (Klotzsch) Lloyd are two infrequently collected stalked gasteroid in Chile. They inhabit arid regions and emerge after early spring rains. New localities and descriptions are presented here.

les clasifica actualmente en la Clase Agaricomycetes. (Gabel \& Gabel 2011); (Mycobank); (Hibbett et al. 1997).

Los hongos gasteroides leñosos desertícolas, clasificados en la familia Agaricaceae, se encuentran en ambientes áridos y semiáridos. El basidioma se desarrolla bajo la superficie del suelo y cuando está maduro emerge por elongación del estípite. Las esporas son liberadas a través de un 
Nuevos registros de hongos gasteroides leñosos desertícolas (Agaricaceae) - Jirón C.

ostiolo apical o por fragmentación del saco esporífero. (Bailey \& Bailey 2003). Este grupo se caracteriza por sus cuerpos fructíferos epigeos en la madurez, con una cabeza fértil sobre el ápice de un estípite bien desarrollado que puede tener una estructura basal, la volva; un peridio consistente de dos capas cuya dehiscencia ocurre por una abertura más o menos irregular, un ostíolo apical, varios poros o una dehiscencia circuncisa; gleba pulverulenta dentro de una cavidad general, consistente de un capilicio (con excepciones), esporas ornamentadas o lisas y ocasionalmente eláteres presentes, basidios distribuidos homogéneamente dentro de la gleba pero desapareciendo en la madurez, aunque persisten en algunos géneros como pequeños grupos. (Moreno, G. et al. 1995). Dentro de esta familia se ubican B. stevenii (Liboschitz) Fr. y $\boldsymbol{C}$. meyenianus (Klotzsch) Lloyd, de distribución cosmopolita pero escasamente colectados en Chile.

En este trabajo se reporta el hallazgo de estos hongos, muy raros, poco frecuentes, escasos y difíciles de encontrar por el hábitat desértico en el que habitan. Las nieblas matinales y ocasionales lluvias permiten que el desierto florezca y también fructifiquen este tipo de hongos.

\section{MATERIALES Y MÉTODOS}

El material biológico de $\boldsymbol{B}$. stevenii corresponde a un ejemplar único colectado en la localidad de Puerto Oscuro, Región de Coquimbo a 59 m.s.n.m. Se encontraba postrado sobre hojarasca en la base de un eucalipto, cerca de una cabaña de veraneo y rodeado de Loasa tricolor. El lugar está influenciado por la neblina costera.

El material biológico de $\boldsymbol{C}$. meyenianus corresponde a una colecta de 5 ejemplares de un grupo de 20 carpóforos emergentes de un suelo arenoso y pedregoso en la localidad de Los Cristales, Región de Atacama, a 899 m.s.n.m. Lugar cercano a la carretera e influenciado por las escasas lluvias del sector. La flora presente en los alrede- dores corresponde a Tropaeolum kingii, Balsamocarpon brevifolium, Lycium stenophyllum, Lycium bridgesii, Proustia ilicifolia, Adesmia argentea, Gutierrezia gayana, Heliotropium sinuatum, Ephedra sp, Caesalpinia angulata, Cistanthe calycina, Bulnesia chilensis, Cordia decandra.

Para el estudio microscópico de ambos materiales se empleó Hidróxido de Potasio 3\% y Azul de algodón con lactofenol. Las microfotografías fueron tomadas con Digital Microscope Imager Suite 2.0.

Todos los ejemplares colectados quedaron depositados en el Museo Nacional de Historia Natural, Sección Botánica, Chile. (SGO).

\section{RESULTADOS Y DISCUSIÓN}

Battarrea stevenii (Liboschitz) Fr. Syst. Mycol. 3: 71829

Basiónimo: Dendromyces stevenii Libosch. Beschreibung eines neu entdecktes Pilzes: 4, t. 1 (1814). Battarrea phalloides var. stevenii (Libosch.) Cleland \& Cheel, Journal of Proceedings of the Royal Society of New South Wales 50: 111 (1916).

Distribución: Se trata de una especie cosmopolita presente en África, América, Europa y Oceanía. (Madrid 2007).

El carpóforo del ejemplar colectado mide $25 \mathrm{~cm}$ de alto. El estípite es café, cilíndrico, leñoso, fibroso y desflecado, más angosto en la inserción del saco esporífero. En la base se inserta dentro de la volva que tiene dos componentes, uno externo coriáceo blanquecino y otro con escamas café. Ambos claramente separados. El saco esporífero, de 3,5 cm de ancho x 1,5 cm de alto, está incompleto debido a una ruptura circuncisa del peridio que eliminó el exoperidio y parte del endoperidio. (Fig. 1 A-C). El endoperidio restante tiene consistencia membranosa, color blanquecino y está formado por hifas entrecruzadas gruesas y delgadas de 1,0-5,0 $\mu \mathrm{m}$ de ancho. De la gleba 


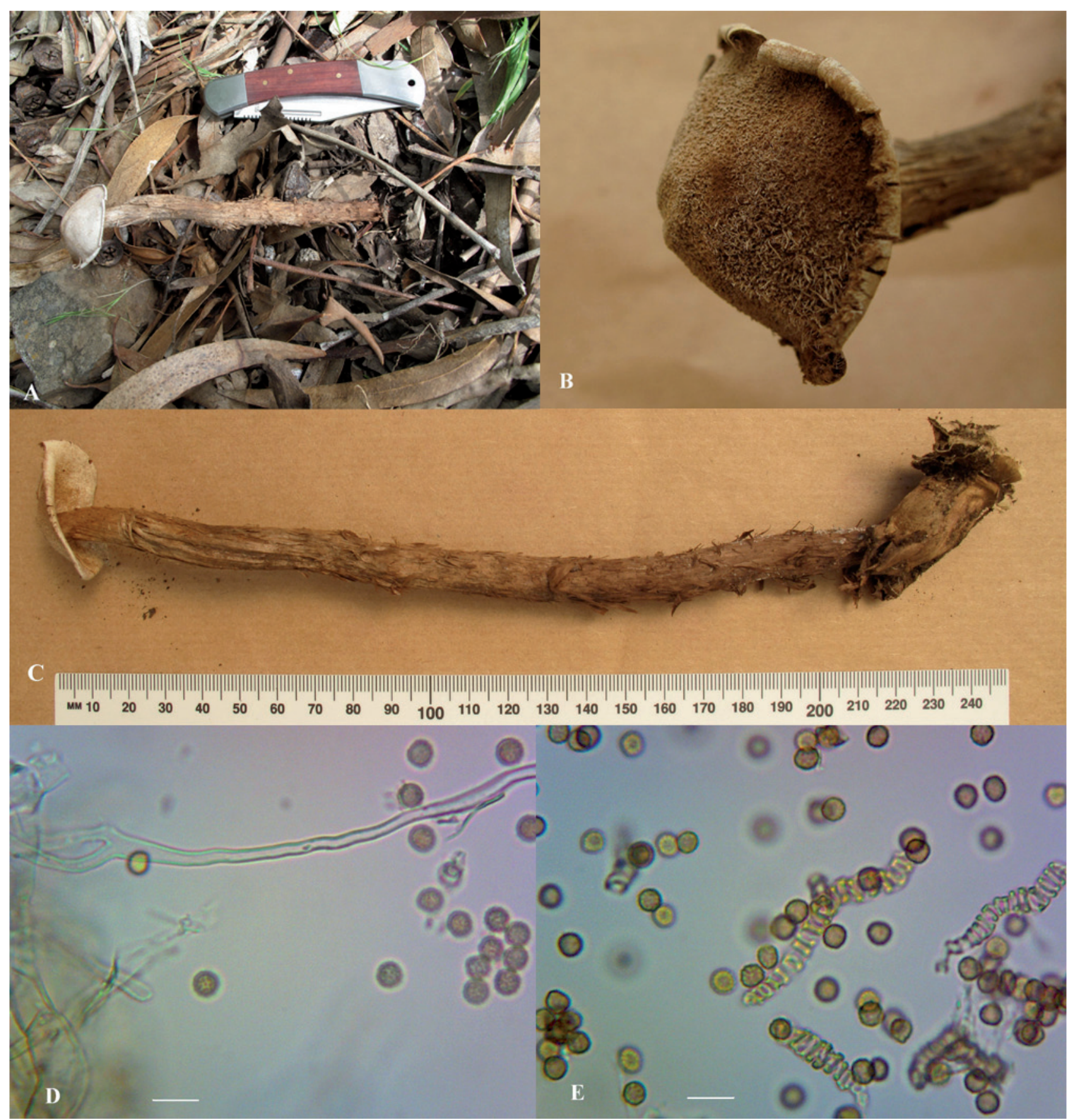

Figura 1. A. Hábitat de B. stevenii. B. Endoperidio con restos de gleba. C. Tamaño del hongo. D. Hifas del capilicio (600X). E. Eláteres y esporas. (600X). D-E Barra $=10 \mu \mathrm{m}$. Fotografías C. Jirón.

Figure 1. A. Habit of B. stevenii. B. Endoperidium with remains of gleba. C. Fungi size. D. Hyphae of capillitium. (600X) E. Elaters and spores (600X). D-E Scale $=10 \mu \mathrm{m}$. Photographs by C. Jirón. 
quedan restos fibrosos blanquecinos y pardos y la observación microscópica muestra 2 componentes: hifas hialinas de 2,5-7,0 $\mu \mathrm{m}$ de ancho, dispuestas en paralelo y eláteres de forma espiralada, paredes gruesas, amarillo-pardo y miden de 2,5-5,0 $\mu \mathrm{m}$ de ancho y el largo es muy variable desde $20-50 \mu \mathrm{m}$ de largo. Las esporas son globosas, con la superficie verrugosa, pardas, de 5,0 $\mu \mathrm{m}$ (Fig. 1 D-E)

Material estudiado: CHILE. Región de Coquimbo, Provincia de Choapa, Puerto Oscuro, $31^{\circ} 25^{\prime}$ S $71^{\circ} 35^{\prime}$ O, 59 m.s.n.m. 12-X-2015. Jirón. (SGO 166586).

Lugares de Colectas de B. stevenii (Libosch.) Fr. en Chile:

Aguada de Tongoy, Huasco. Región de Atacama. (Furci 2011).

Paposo, cerro el Mirador (24 59 $59^{\circ} \mathrm{S} ; 70^{\circ} 26^{\circ} \mathrm{O}$ ), Región de Antofagasta, (Madrid 2007).

Parque Nacional Fray Jorge, Región de Coquimbo (Lazo 1991).

Chlamydopus meyenianus (Klotzsch) Lloyd Mycol. Writings 1: 134-135 1903.

Basiónimo: Tylostoma meyenianum Klotzsch Fungi a Meyen coll. P. 243 t. V, f 4.

Tulostoma meyenianus Klotzsch Nov. Act. Caes. Leop. Carol. Nat. Cur. 19: 2431843.

Tulostoma deserticola Philippi, R. Florula atacamensis p. 1301860.

Distribución: América del Norte, América del Sur, Norte de África y Australia (Long, W. H., \& Stouffer, D. J. (1946).

El carpóforo de los ejemplares colectados fluctúa entre 11,5 y 14,0 cm de alto. El basidioma está unido al ápice dilatado del estípite y consta de un exoperidio, un endoperidio y la gleba. El saco esporífero es subgloboso, deprimido, de 3,0-3,5 $\mathrm{cm}$ de ancho x 1,5-2,0 cm de alto; de consisten- cia coriácea, color blanquecino, con dehiscencia por una apertura apical de bordes irregulares o por fragmentación de las paredes. (Fig. 2 A-C). El exoperidio, presente en 4 de los ejemplares, tiene consistencia suave en la parte interna y verrugosa e impregnada de arena en la parte externa. Compuesto de hifas hialinas, entremezcladas, algunas muy finas y otras globosas de $8-25 \mu \mathrm{m}$ de ancho x 45-70 $\mu \mathrm{m}$ de largo. El endoperidio está formado por una mezcla compacta de hifas hialinas de 2,5$3,8 \mu \mathrm{m}$ de ancho. La gleba es muy pulverulenta de color ferruginoso y formada por hifas, basidios en fascículos y esporas. Las hifas son hialinas, delgadas y gruesas, entre 3,5-10.0 $\mu \mathrm{m}$ de ancho; las más delgadas con algunas fíbulas y las más gruesas son densas, están ramificadas y sostienen los fascículos de basidios. Las esporas son globosas, verrugosas, de 5,0-7,0 $\mu \mathrm{m}$. de diámetro. (Fig. $2 \mathrm{D}-\mathrm{F}$ ).

El estípite, de 5,5-12 cm de alto, generalmente curvo en los ejemplares más jóvenes, de $0,6-1,0 \mathrm{~cm}$ de ancho en la base y $1,5-2,0 \mathrm{~cm}$ en el ápice. Consistencia leñosa, lacerado, fibrosa, con estrías longitudinales, color blanquecino-sedoso, cilíndrico en la base y aplastado en el ápice donde se une al saco esporífero. En la base se une a la volva en forma de copa de 1,5-2,0 cm de alto y 2,3-3,0 $\mathrm{cm}$ de ancho, cubierta externamente de arena.

Material estudiado: CHILE, Región de Atacama, Provincia de Huasco, Los Cristales, 29 $19^{\circ}$ '20" S $71^{\circ} 01^{\prime} 17^{\prime \prime}$ O, Ruta 5 Norte, Km 577 oeste, 899 m.s.n.m., 14-IX-2015. Jirón \& von Harpe. (SGO 166587 al 166591).

\section{Lugares de colectas de C. meyenianus (Klotzsch) Lloyd, en Chile:}

Entre Cachinal de la Sierra y Agua del Profeta $\left(24^{\circ}\right.$ 54'S 69 07’ O) elevación 9000 ft., descrito como Tulostoma deserticola (Philippi 1860).

Quebrada del Inca entre Ollagüe y Amincha, en

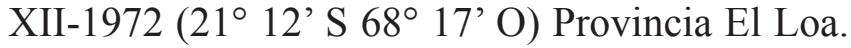
Región de Antofagasta. (Mahú, 1980). 


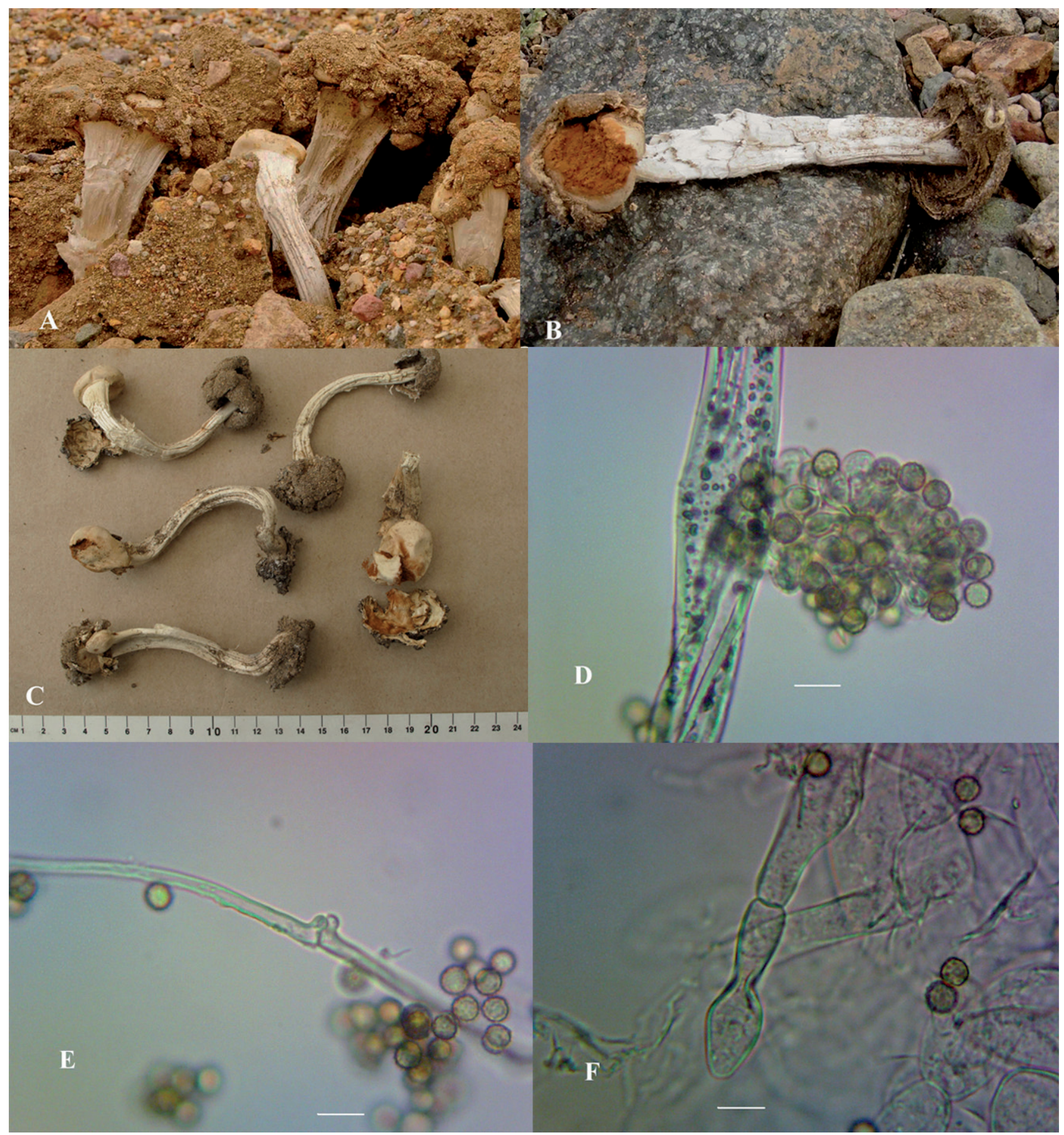

Figura 2. A y B. Hábitat de C. meyenianus. C. Tamaño de los hongos. D. Basidios en fascículos. (600X). E. Fíbula. F. Hifas globosas del exoperidio. D-F: Barra $=10 \mu \mathrm{m}$. Fotografías: von Harpe \& Jirón.

Figure 2. $\boldsymbol{A}$ and $\boldsymbol{B}$. Habit of $\boldsymbol{C}$. meyenianus. C. Fungi size. D. Fasciculate basidia. (600X). E. Clamp connections. F. Exoperidial hyphae inflate. D-F: Scale $=10 \mu \mathrm{m}$. Photographs by von Harpe \& Jirón. 


\section{AGRADECIMIENTOS}

A la Fundación Philippi de Estudios Naturales por su apoyo en este estudio. A las asistentes, Karen Lozano y Walezka Zamorano, del Laboratorio de Microbiología de la Universidad Tecno- lógica Metropolitana, por colaborar en el análisis microscópico de los hongos. A Rudy Iguaín por la revisión del manuscrito.

\section{REFERENCIAS}

Bailey, L.; M. Bailey. (2003). Trial field key to the woody desert fungi found in the Pacific Northwest. Pacific Northwest Key Council.

Furci, G. (2013). Guía de Campo: Hongos de Chile. OFundación Fungi y Giuliana María Furci George-Nacimiento.

Gabel, A. C.; M.L. Gabel (2011). New records of gasteroid and secotioid fungi from sand dunes in northwestern South Dakota. Proceedings of the South Dakota Academy of Science, Vol. 90.

Hibbett, D. et al. (1997). Evolution of gilled mushrooms and puffballs inferred from ribosomal DNA sequences. Proc. Natl. Acad. Sci. USA. Vol. 94, pp. 12002-12006

Lazo, Waldo. (1991). Notas micológicas y bacteriológicas: basidiomicetes y streptomycetes de Chile. Bol. Micol. 6: 41-42

Long, W. H., \& Stouffer, D. J. (1946). Studies in the Gasteromycetes XIV the Genus Chlamydopus. Mycologia, 38(6), 619-629. http://doi. org/10.2307/3755302
Madrid. H. 2007. Battarrea stevenii (Liboschitz) Fr. En Paposo II Región de Chile. Boletín Micológico Vol. 22: 37-39 2007

Mahú, M. 1980. New collection and new locality in Chile for Chlamydopus meyenianus

(Klotzsch) Lloyd (Gasteromycetes). Mycotaxon Vol. $11 \mathrm{~N}^{\circ} 2$

MycoBank, official nomenclatural repository for fungi. http://www.mycobank.org

Moreno, G.; A. Altés; C. Ochoa and E. Wright. Contribution to the Study of the Tulostomataceae in Baja California, México. Mycologia Vol. $87 \mathrm{~N}^{\circ}$ 1 (Jan.-Feb. 1995) pp.96-120

Norvell, L.L.; J.F. Ammirati and S.A. Redhead. 2008. Woody desert puffballs of the Pacific Northwest 1: Chlamydopus meyenianus. North American Fungi 3(7): 127-138. http://dx.doi. org/10.2509/naf2008.003.0076

Philippi, R. A. (1860). Viaje al Desierto de Atacama. 2a Ed. Biblioteca Fundamentos De La Construcción de Chile. Diciembre 2008. 